\title{
Implementing a Communities of Practice model to enhance learning and teaching at a transnational university in China
}

Henk Huijser, James Wilson, Dawn Johnson, Jianmei Xie

Xi'an Jiaotong-Liverpool University (XJTLU)

\section{Introduction}

In this paper, we present a case study of a communities of practice (CoPs) model (Lave and Wenger, 1998) to enhance learning and teaching at a transnational university in China. "Communities of practice are groups of people who share a concern or a passion for something they do and learn how to do it better as they interact regularly" (Wenger \& Wenger-Trayner, 2015). A CoPs model was chosen for two main reasons: firstly, to suit a highly diverse organisational structure, in which different academic departments have diverse needs and characteristics, making it difficult to apply a 'one-size-fits-all' model; secondly, to allow the Educational Developers in the central unit, called Academic Enhancement Centre (AEC), to tailor their learning and teaching support to such diverse needs. Furthermore, the CoPs model was chosen as an alternative to a centrally-designed workshop model, in which academic staff attend a series of workshops on offer and have their attendance credited. A CoPs model was seen as a potentially more organic and 'bottom-up' approach to professional development and learning, as opposed to a more centralised and 'top-down' approach. Of course, there are different degrees to this binary and we do not intend to argue for one or the other. Rather, the intention is to implement a CoPs model that complements other forms of professional learning at the university.

This case study outlines the rationale behind this approach at Xi'an Jiaotong-Liverpool University (XJTLU), a joint venture between Xi'an Jiaotong University in China and the University of Liverpool in the UK, characterised by a diverse international academic staff. It further reports on the early stages of implementation and discusses some of the initial challenges encountered. We draw on Wenger and Wenger-Trayner's (2016) 'value creation framework' to argue that, while traditional forms of professional development often stay couched in potential value, a CoPs model promises to move professional learning beyond immediate and potential value towards applied, realised, strategic, enabling and ultimately transformative value (Wenger, Trayner and de Laat, 2011).

\section{Organisational context - Xi’an Jiaotong-Liverpool University}

According to its vision, Xi'an Jiaotong-Liverpool University (XJTLU) is a research-led international university, based in Suzhou, Jiangsu Province, China. The university was jointly founded in 2006 by Xi'an Jiaotong University (China) and the University of Liverpool (UK) and it opened its doors to about 160 students in September of the same year. As an independent Sino-Foreign cooperative university, XJTLU captures the essence of both prestigious parent universities and was the first one of its kind to be approved by the Ministry of Education in China. Currently, it has circa 10,000 registered students on over fifty undergraduate and postgraduate degree programmes. Since XJTLU is an English as a Medium of Instruction (EMI) 
University, the majority of its degree programmes are delivered in the English language, with exceptions for those modules required as part of the Chinese degree.

As this is a research-intensive university, learning and teaching processes tend to be juxtaposed with research and not treated as of equal worth and are thus often regarded as secondary issues by the large majority of faculties, which tend to be primarily concerned with developing their discipline-based research profiles. There is thus minimal evidence of learning and teaching communities across the University. The AEC development programmes are seen by many as a requirement, rather than as an opportunity to explore learning and teaching pedagogies and methodologies with a view to improving the quality of teaching and, as a result, the learning experience of students. Within such a context, the adoption of a CoPs approach, the better to support professional development and learning within an informal discursive setting at departmental level, faces a considerable challenge in that it may be viewed sceptically by many Heads of Departments as 'just another meeting to attend'. Furthermore, XJTLU is a transnational university with a large influx of international staff from wide-ranging educational backgrounds and environments who bring with them very varied preconceptions about learning and teaching, those quite often misconceived or misunderstood in relation to the cross-cultural context in China, which presents some unique challenges (Jin and Cortazzi, 2006). Moreover, in the Chinese higher education context, there is little evidence of CoPs being used before now, even if there they have been trialled in other professional environments (Hasmath and Hsu, 2015; Zhang and Watts, 2008).

In an attempt to foster a better understanding of the importance of learning and teaching and CoPs across the university, the AEC decided, as a starting point, to make CoPs, and social learning more broadly, the primary focus of the Annual Learning and Teaching Colloquium, which was expected to engage senior management simultaneously in the process. Ultimately, the goal was to develop a culture of continuous organisational learning.

\section{A communities of practice model as a pedagogical approach to organisational learning}

On the surface, a CoP seems like a straightforward concept, but this may be both a strength and a weakness, with some readily grasping its nature and others oversimplifying it as a term suitable for any group of people getting together. It is not quite as simple as the latter. The concept was originally coined by Lave and Wenger (1991) who positioned learning as "an integral and inseparable part of social practice" (p. 31), implying that CoPs form spontaneously in organisations and cannot be 'created' from the top by management. The 'practice' element of a CoP is a crucial part of the concept and ensures that the learning is 'situated' (Farnsworth, Kleanthous and Wenger, 2016). In Sense's (2015) words, "situated learning actually evolves (explicitly or implicitly) through the learning processes of observation, dialogue, storytelling and conversations between people as they participate and interact within a practice, and can be considered in more pragmatic terms as learning-on-the-job" (p. 288). The emphasis on 'situated learning' means that no two CoPs are the same and that they invite differing levels of participation that evolve over time as relationships are developed and strengthened (Gullick and West, 2016). In terms of organisational learning, CoPs are thus about situated learning, 
mentorship, and building capacity (Wenger, McDermott and Snyder, 2002). From the point of view of the Academic Enhancement Centre at XJTLU, this raises the question: Can CoPs be 'created' in a structured manner as part of an organisational professional development strategy?

As Macpherson and Antonacopoulou (2013) note, "CoP was not originally intended to describe the formulation of groups that management creates, but that does not mean management cannot support an environment in which they might flourish" (p. 267). Similarly, Sense (2015) draws attention to the intentionality of establishing what he calls a 'CoP vessel' to "help stimulate and facilitate social learning" (p. 287), whereby 'intentionality' refers to a deliberate structural approach to organisational learning with CoPs as the vehicle. This is a crucial element and draws attention to the idea that it is necessary for "[CoPs'] formation and persistence that appropriate stimuli and conditions and resources are established to support [their] development" (Sense, 2015, p. 289). If CoPs are to thrive, the onus is therefore, to some extent, on management to provide such conditions and resources and a central unit, such as the Academic Enhancement Centre at XJTLU, can play a potentially important role in liaising with senior management on the one hand and academic staff on the other. However, as Macpherson and Antonacopoulou (2013) rightly point out, "the strategic adoption of CoPs is unlikely to go uncontested and they may not operate as intended, in practice" (p. 266). The case study of the initial establishment of CoPs at XJTLU is making this very clear. However, this is precisely the point of an approach to continuous organisational learning that deliberately steps away from a centralised one-size-fits-all approach. Contestation in this context is considered healthy rather than problematic.

In stepping away from a centralised, workshop-based approach, Wenger and Wenger-Trayner's (2016) 'value creation framework' is considered to help address the issue of 'value' and to provide a framework to demonstrate the potential value of a particular CoP or whether it 'works'. The framework has seven different values: 1) immediate value - immediate experience while engaging with the community; 2) potential value - what the learning of the community potentially produces; 3 ) applied value - practical application of the learning; 4) realised value - the difference the community is making; 5) transformative value - the learning of the community is itself transformed through continuous evaluative cycles; 6) strategic value - ability of the community to engage in strategic conversations to get what it needs from management; 7 ) enabling value - support processes that make the community's life possible. The value creation framework includes four evaluative key questions for each of the seven values, covering 'aspirations' (What would the ideal look like?), 'conditions' (What would the ideal conditions be?), 'indicators' (What indicators should be used for success?) and 'data' (What kind of data is needed to show that success is achieved). From our point of view, the attraction of this framework is that it gives us an instrument to demonstrate value to those who would need to support CoPs at XJTLU.

\section{Creating the conditions for the establishment of communities of practice at XJTLU}

The original conceptualisation of CoPs included three key concepts: identity, participation and governance (Lave and Wenger, 1991). In terms of participation, Macpherson and 
Antonacopoulou (2013) argue that "the deployment of systems and structures by strategic management sets the landscape on which participation occurs" (p. 269). Since its early conceptualisation, Wenger et al (2002, p. 13) have further developed seven principles for fostering CoPs' energy and internal direction: 1) designing for evolution, 2) opening dialogue between insider and outsider perspectives, 3) inviting differing levels of participation, 4) developing public and private spaces, 5) focusing on value, 6) combining familiarity with excitement and 7) creating a rhythm for the community. From an implementation point of view, these principles help to keep us, as Educational Developers at XJTLU, focused on the cultivation of CoPs, and draw attention to AEC's role of fostering CoPs, rather than directing them. The CoPs that have been established so far (which will be discussed in the next section) show this very clearly, as they are very diverse, but are developed from the bottom up, a process fostered by AEC.

XJTLU's strategy for the implementation of CoPs was based on a desire by AEC to develop a more structural approach to supporting learning and teaching at XJTLU and to support the implementation of an XJTLU Continuing Professional Development (CPD) framework. The fundamental element of the strategy was the establishment of cluster-based ${ }^{1} \mathrm{CoPs}$, which would be driven by 'CoP Cluster Leads', and facilitated by an AEC Educational Developer. As they would be 'embedded' in clusters, rather than represented in a hierarchical manner, the CoPs could then function as an opportunity for the AEC, through its Educational Developers, to identify and disseminate, in a structured manner, good learning and teaching practice.

The strategy was designed to address three key concerns:

1. that, excluding the Certificate in Professional Studies (CPS) programme and the Postgraduate Research Skills Development (PGR) programme, educational development in general is currently too sporadic and reactive, rather than structural and proactive;

2. that there are limited opportunities for 'bottom-up' ownership of, and input into, learning and teaching;

3. that it is currently difficult to track progress when it comes to good practice in learning and teaching.

The suggestion was to establish a CoP in each cluster: six in total, plus one in the Language Centre.

The CoPs would function in the following manner:

- Each CoP would be driven by a Cluster Lead (appointed by the Cluster Head, based on expressions of interest);

- Membership of each CoP would consist of the Cluster Lead, the AEC Educational Developer and all lecturers/teachers who wanted to join;

\footnotetext{
${ }^{1}$ XJTLU does not use the term 'Faculties', but instead uses discipline-based Departments that are grouped into 'Clusters', which are very similar to Faculties at other universities.
} 
- They would meet once a month for two hours to discuss a particular learning and teaching theme and follow a 'domain', 'practice', 'community' pattern (Wenger and Wenger-Trayner, 2015; McDonald, 2012);

- Each would function as a CoP (NOT a formal committee);

- Each would be facilitated initially by an AEC Educational Developer;

- Each month, a learning and teaching related theme would be identified for the next session;

- This would be a 'bottom-up' process, but Educational Developers would be able to communicate and incorporate 'top-down' requirements;

- Good practice would be both identified during the CoPs and expected to emerge from the CoPs. The dissemination of this practice would follow existing XJTLU structures, e.g. Learning and Teaching Colloquium, Teaching Development Fund (TDF), Teaching Conference Fund (TCF), the CPS Programme, the PGR Programme (which included professional development for Teaching Assistants), etc.

Initially, CoPs were introduced in the form of a specific CPS workshop, which was followed by the Annual Learning and Teaching Colloquium soon after. As noted above, to launch the process, CoPs were a key focal point of this Colloquium in 2016, which featured a number of workshops and a keynote address/seminar by Etienne Wenger and Beverly Wenger-Trayner. This was supported by senior management to build initial momentum for the implementation process. In addition, this was particularly important in an institutional context where staff come from a wide variety of different national contexts, creating the potential for very different understandings of what a CoP might be or how it might function. The Colloquium was therefore seen as an opportunity to develop a shared understanding about the meaning and application of CoPs.

Building on this momentum, XJTLU plans to foster the establishment of cluster-based CoPs, a process that is now under way.

\section{Evaluating the start-ups - an initial evaluation using the Value Creation Framework}

Heads of Department (HoDs) were initially asked by AEC to identify key people in their clusters who could act as potential 'leads' for the development of CoPs within different academic disciplines here at XJTLU. This was a difficult balancing act, in that it created an immediate tension between 'top-down' decision making, and 'bottom-up' organic development. However, at this early stage, we made the strategic decision that it was important for HoDs to be involved in the process rather than alienated from it, as space needed to be created for the 'bottom-up' process to occur, paradoxical as that may sound. The first call for engagement produced four potential leads and the Educational Developers within AEC arranged to meet with the named persons in order to explore/support the possibility of encouraging people to come together to discuss shared challenges they had within their own particular contexts. Follow-up conversations have revealed that all four of these identified leads are now at different stages of development of their CoPs. Confirmation of similarities and differences of experience was not 
unexpected and highlighted the multitude of potential constraints and limitations of such an approach.

While one Cluster Lead has had some initial success with getting colleagues and peers together to explore the development of a CoP within their own context, another has struggled to do so. Yet another has made a first attempt to develop a cross-disciplinary CoP in his Cluster. AEC helped to facilitate the initial CoP sessions, but we allowed the extent of our involvement to depend on the Cluster Leads, so AEC's involvement varied between hands-on training and mentorship and light-touch facilitation. Using the 'Value Creation Framework', we have been able to evaluate the 'start-up' projects/experiences.

The immediate value of setting up CoPs was predominately viewed as a way of better understanding current cross-discipline methodology and of sharing/discovering good practice, in terms of what works/does not work in individual situations. Within the Language Centre at XJTLU, agreement was reached about the conditions needed for this venture to be successful and face-to-face meetings for two hours each month were decided upon. The attendance at the first meeting was healthy and the conversations exhibited energy and engagement between members. This engagement translated into an agreement by a number of members to take ownership of one of the identified issues of concern to the community (e.g. student engagement, or lack thereof; communication within and across departments); other members agreed to support them. One of the constraints that emerged was the timing of this first meeting and, upon reflection, the lead person realised that this was a very busy time for colleagues in their work schedule. This may explain why follow-up conversations have been minimal, as heavy teaching workloads present a significant challenge to the building of momentum.

The potential value of these CoPs is the aspiration to discover new approaches to dealing with the challenges faced in everyday practice and to apply new ways of doing things to enhance teaching and thus the learning experiences of students. Requests for implementation assistance from AEC were sought and provided, but it is not the intention of AEC to be the drivers or maintainers of these groups, as this would ultimately be unsustainable and ineffective. Action planning and long-term implementation should be driven from within the community itself. However, in order for this to be successful, acknowledgment and support from senior managers within each cluster and allocation of time within staff workloads (Sense, 2015) for these activities to take place are essential. In order to enable cross-fertilisation across XJTLU at a more strategic level, acknowledgement in the form of a regular agenda item for feedback on progress should be built into the regular University Learning and Teaching Committee structure.

Perhaps the most important lesson learned overall was the amount of support that the lead person needs in order to carry out her/his role. This applies in particular to the initial stage. The lead person for the Science Cluster, for example, has requested financial support for the initial CoP meeting, so that refreshments can be served as part of establishing a semi-informal environment. Interestingly, this cluster lead has also explicitly requested AEC members not to be present at this initial meeting so as not to create the impression that it is an initiative driven 'from above'. For those cluster leads that struggle to build momentum, an organically-occurring solution has been the development of a CoP for all leads, exploring common challenges that 
they have encountered and allowing them to share different approaches to some common problems. It is crucial that these leads feel tangible support in carrying out their role.

Whilst there is definitely some palpable initial optimism and excitement about the CoP approach, there are some significant challenges to be addressed, which include:

- the transitory nature of staff at XJTLU, which is a challenge to continuity and consistency;

- the lack of reward and recognition for investing time and effort in this initiative, especially in the case of Cluster Leads.

Some other factors that have been identified as important in the sustainability of both individual CoPs and the overall CoP approach at XJTLU include:

- the need for encouragement to develop and sustain feedback loops within the process (This should include a clear evaluation process to ascertain why CoPs work in some clusters and not in others and subsequently to develop ways of encouraging participation and develop appropriate support.);

- the acknowledgement that there are other CoPs happening quite informally across XJTLU, whether or not they are labelled as such by AEC (The work of the AEC team may be to identify such groups and encourage them to create a feedback loop for the teaching and learning enhancement work they are already involved with, so that we may have a fuller picture of CoP activity across XJTLU.);

- a regular CoPs feature within the AEC e-Newsletter, as a way of disseminating and nurturing this new way of working at XJTLU.

\section{Conclusion}

Overall, while there are some encouraging initial signs that the CoP approach may develop into a sustainable organisational learning culture at XJTLU, there are significant challenges ahead. The potential lies in moving beyond the initial two stages ('immediate' and 'potential' value) of Wenger et als (2011) value creation framework. The expectation and hope at XJTLU is that, once they are fully established and functioning, the various CoPs, whatever discipline- or cluster-based form they take, will be able to deliver applied, realised, strategic, enabling, and ultimately transformative value. Educational development will then be a matter of 'guiding on the side' towards continuous improvement in learning and teaching in a challenging transnational higher education environment.

\section{Reference list}

Farnsworth, V., Kleanthous, I. and Wenger, E. (2016) 'Communities of practice as a social theory of learning: a conversation with Etienne Wenger.' British Journal of Educational Studies, 64(2), 139-160.

Gullick, J. and West, S. (2016) 'Building research capacity and productivity among advanced 
practice nurses: an evaluation of the Community of Practice model.' Journal of Advanced Nursing, 72(3), 605-619.

Hasmath, R. and Hsu, J. (2015) 'Communities of Practice and the NGO sector in China.' Paper presented at the Association for Research on Nonprofit Organizations and Voluntary Action Annual Conference, Chicago, 19-21 November.

Jin, L. and Cortazzi, M. (2006) 'Changing practices in Chinese cultures of learning.' Language, Culture \& Curriculum, 19(1), 5-20.

Lave, J. and Wenger, E. (1991) Situated learning: Legitimate peripheral participation. Cambridge: Cambridge University Press.

Macpherson, A. and Antonacopoulou, E. (2013) 'Translating strategy into practice: the role of communities of practice.' Journal of Strategy and Management, 6(3), 256-285.

McDonald, J. (2012) Community, domain, practice: facilitator catch cry for revitalising learning and teaching through communities of practice (Project Report). Sydney: Australian Learning and Teaching Council.

Sense, A. (2015). 'Work-based researchers and Community of Practice: Conceptual and gestational dilemmas.' Australian Journal of Adult Learning, 55(2), 283-308.

Wenger, E. and Wenger-Trayner, B. (2016) 'Planning and assessing the value(s) created by learning in communities.' Seminar presented at XJTLU's Annual Learning and Teaching Colloquium 2016: Imagining the Next 10 years in Learning and Teaching. Suzhou, China, 7-8 April.

Wenger, E. and Wenger-Trayner, B. (2015) 'Introduction to communities of practice: a brief overview of the concept and its uses.' Available at: http://wenger-trayner.com/introduction-tocommunities-of-practice/ (Accessed: 25 April 2016).

Wenger, E., McDermott, R. and Snyder, W. (2002) Cultivating communities of practice. Boston, MA: Harvard Business School Press.

Wenger, E., Trayner, B. and de Laat, M. (2011) Promoting and assessing value creation in communities and networks: a conceptual framework. (Rapport 18). Heerlen: Open University of the Netherlands. Available at: http://wenger-trayner.com/resources/publications/evaluationframework/ (Accessed: 26 April 2016).

Zhang, W. and Watts, S. (2008) 'Online communities as communities of practice: a case study.' Journal of Knowledge Management, 12(4), 55-71. 